\title{
Singular solutions of a non-holonomic system as limits separating solution groups of particular type
}

\author{
Jiř́ Náprstek $^{1, *}$ and Cyril Fischer ${ }^{1, * *}$ \\ ${ }^{1}$ Inst. of Theoretical and Applied Mechanics, CAS, Prosecká 76, CZ-190 00 Prague, Czech Republic
}

\begin{abstract}
The non-holonomic system represents a model of the ball tuned mass damper (TMD), as used to absorb vibration of selected engineering structures, where conventional absorber types are inapplicable. The device consists of a ball moving within a spherical cavity fixed with the structure. To deduce a governing differential system the Appel-Gibbs formulation has been employed. The non-linear mathematical model includes six degrees of freedom and three non-holonomic constraints. The system has an auto-parametric character. The homogeneous differential system in the normal form is formulated. Its general properties are investigated for various settings of non-homogeneous initial conditions. Several singular solutions are extracted and physically interpreted. In principal, they represent limits separating solution groups of a certain character. The shape and general character of regular solutions within individual domains staked out by these limits are analyzed in order to facilitate a practical application of this theoretical background.
\end{abstract}

\section{Introduction}

The study is motivated as a theoretical background of a passive vibration absorber of a ball type. The basic principle comes out of a rolling movement of a metallic ball of a radius $r$ inside of a rubber coated cavity of a radius $R>r$. This system is closed in an airtight case and installed at the top of the served structure. First papers dealing with the theory and practical aspects of ball absorbers have been published during the last decade, see [1]. The ball type absorber proved very well being used at slender structures exposed to wind excitation, e.g. TV towers, guyed masts, etc. Although widely used conventional pendulum type absorbers are very effective and reliable, they have several disadvantages limiting their application. These shortcomings can be avoided using the absorber of ball type.

Hamiltonian functional and relevant Lagrange equation system are popular tools in investigation of dynamic systems. Various generalizations allow to extend the class of problems concerned slightly beyond conventional limits of Hamiltonian system. This strategy is very effective, particularly concerning 2D and simpler 3D systems. However, the governing differential systems of most non-holonomic 3D systems suffer from inadequate complexity, when deduced using this way. Any analytical investigation of such a governing system is rather impossible and its physical interpretation can be multivalent. For easier analysis, particularly of systems with non-holonomic constraints, the Appell-Gibbs approach seems to be more effective providing more transparent governing systems. For more information, see monographs e.g. [2], [3], or journal papers e.g. [4], [5] and many others.

\footnotetext{
*e-mail: naprstek@itam.cas.cz

**e-mail: fischerc@itam.cas.cz
} 
The problem of dynamics of such a system is very complex and it would be beyond limits of this paper to treat all its aspects. Authors discussed a number of its attributes for instance in [6], [7] and in other publications, where more references can be found. In this article we concentrate on one important effect stemming from a strongly nonlinear character of the system. It reveals that the governing differential system in the homogeneous case (without external excitation) exhibits singular solutions which can be considered as limits separating regular solutions into certain groups according to their individual properties. These singular solutions are characterized by nonzero initial conditions and correspond to the case when the ball starts its movement from unbalanced initial condition in the fixed cavity. Anyway, these singular solutions do not create any separable space from a strict mathematical viewpoint, but still they can be useful tool for a certain style of classification. Authors admit that except singular solutions discussed in the following text, there can exist more singular solutions of the system analyzed. However, these singular solutions do not seem to be important from the viewpoint of separation of regular solutions.

\section{Appell-Gibbs approach}

The basis of the Appell-Gibbs approach is the Appell-Gibbs function (often referred to as an energy acceleration function), which is defined as a function of six component characterizing motion of one stiff body in 3D:

$$
\mathcal{G}=\frac{1}{2} M\left(\ddot{u}_{G x}^{2}+\ddot{u}_{G y}^{2}+\ddot{u}_{G z}^{2}\right)+\frac{1}{2} J\left(\dot{\omega}_{x}^{2}+\dot{\omega}_{y}^{2}+\dot{\omega}_{z}^{2}\right)
$$

where $M$ - mass of the ball, $J$ - central inertia moment of the ball with respect to its center $G$, $\omega$ - angular velocity vector of the ball with respect to its center $G, \mathbf{u}_{G}$ - displacement of the ball center with respect to absolute origin $O, C$ contact point of the ball and cavity, $A$ - moving origin related with the cavity in its bottom point. Coordinates $\mathbf{x}=x, y, z$ - cartesian coordinates with origin in the point $O$.

The function $\mathcal{G}$ allows to write the AppellGibbs differential system:

$$
\begin{aligned}
\partial \mathcal{G}^{r} / \partial \dot{\omega}_{x} & =F_{G x}, \\
\partial \mathcal{G}^{r} / \partial \dot{\omega}_{y} & =F_{G y}, \\
\partial \mathcal{G}^{r} / \partial \dot{\omega}_{z} & =F_{G z},
\end{aligned}
$$

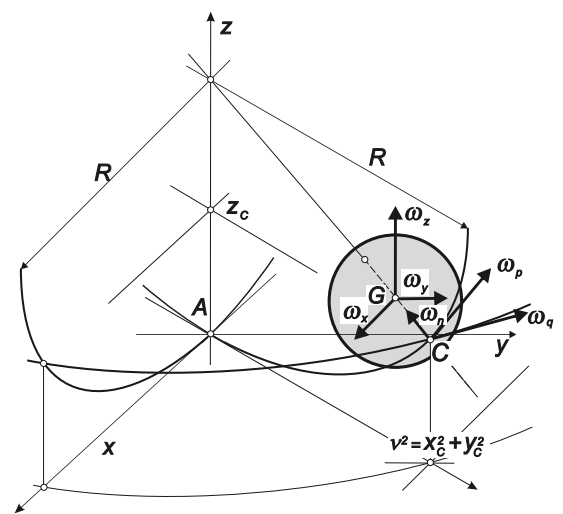

Figure 1. Ball rotation vector in moving coordinates

where $\mathcal{G}^{r}$ is the reduced Appel function where only terms with 2nd time derivatives $\left(\ddot{\mathbf{u}}_{G}, \dot{\omega}\right)$ are kept, $\mathbf{F}_{G}$ - external force vector acting in $G$. Vector $\mathbf{F}_{G}$ can be determined using the virtual displacements principle.

Using Pfaff theorem and adopting a conjecture of non-sliding contact between the ball and cavity the velocities of the ball center with respect to origin can be deduced providing the respective non-holonomic constraints of "perfect" rolling:

$$
\begin{aligned}
& \dot{u}_{G x}=\dot{u}_{A x}+\rho\left(\omega_{y}\left(u_{C z}-R\right)-\omega_{z} u_{C y}\right), \\
& \dot{u}_{G y}=\dot{u}_{A y}+\rho\left(\omega_{z} u_{C x}-\omega_{x}\left(u_{C z}-R\right)\right), \\
& \dot{u}_{G z}=+\rho\left(\omega_{x} u_{C y}-\omega_{y} u_{C x}\right),
\end{aligned}
$$$$
\text { where: } \rho=1-r / R
$$

Constraints Eqs (3) should be differentiated with respect to time in order to substitute into the Appell-Gibbs function Eq. (1). Performing that, the function gets a form $\mathcal{G}=\mathcal{G}_{2}+\mathcal{G}_{1}+\mathcal{G}_{0}$, 
where $\mathcal{G}_{2}, \mathcal{G}_{1}$ and $\mathcal{G}_{0}$ are polynomials of the second, first and zero degree of $\omega$ components. In such a case $\mathcal{G}$ can be replaced by $\mathcal{G}^{r}=\mathcal{G}_{2}+\mathcal{G}_{1}$ as $\mathcal{G}_{0}$ disappears during differentiation with respect to $\dot{\omega}$ components following Eqs (2).

\section{Governing differential system}

Carrying out the differentiation outlined in Eqs (2), it can be written after some adaptations:

$$
\begin{aligned}
& J_{s} \dot{\omega}_{x}=\left(\left(\ddot{u}_{A y}+\rho\left(\omega_{z} \dot{u}_{C x}-\omega_{x} \dot{u}_{C z}\right)\right)\left(u_{C z}-R\right)-\right. \\
&\left.-u_{C y}\left(g+\rho\left(\omega_{x} \dot{u}_{C y}-\omega_{y} \dot{u}_{C x}\right)\right)\right)-D_{G x} / m, \\
& J_{s} \dot{\omega}_{y}=\left(-\left(\ddot{u}_{A x}+\right.\right.\left.\rho\left(\omega_{y} \dot{u}_{C z}-\omega_{z} \dot{u}_{C y}\right)\right)\left(u_{C z}-R\right)+ \\
&\left.+u_{C x}\left(g+\rho\left(\omega_{x} \dot{u}_{C y}-\omega_{y} \dot{u}_{C x}\right)\right)\right)-D_{G y} / m, \\
& J_{s} \dot{\omega}_{z}=\quad\left(\begin{array}{c}
\left(\ddot{u}_{A x}+\rho\left(\omega_{y} \dot{u}_{C z}-\omega_{z} \dot{u}_{C y}\right)\right) u_{C y}- \\
\left.-\left(\ddot{u}_{A y}+\rho\left(\omega_{z} \dot{u}_{C x}-\omega_{x} \dot{u}_{C z}\right)\right) u_{C x}\right)-D_{G z} / m .
\end{array}\right.
\end{aligned}
$$

where: $J_{s}=\left(J+m \rho^{2} R^{2}\right) /\left(m \rho^{2}\right)$ - take a note that $J+m \rho^{2} R^{2}$ represents the mass inertia moment of the ball with respect to the center of the cavity. Terms $D_{G x} / m, D_{G y} / m, D_{G z} / m$ which are linear functions of $\omega_{x}, \omega_{y}, \omega_{z}$ determine the viscous type of the damping. Two types of the damping are considered within the vector $\mathbf{D}_{G}=\left(D_{G x}, D_{G y}, D_{G z}\right)^{T}$, in particular affecting rolling $\left(\kappa_{r}\right)$ and spinning $\left(\kappa_{s}\right)$ movement of the ball. External excitations are specified by movement or acceleration in the point $A$. Because the point $A$ is considered to be fixed in this study, we substitute into Eqs (4): $\ddot{u}_{A x}=\ddot{u}_{A y}=\ddot{u}_{A z}=0$.

In order to obtain the system in the form with first time derivatives concentrated on left side, the first derivatives $\dot{\mathbf{u}}_{C}$ in its right sides should be expressed in displacements $\mathbf{u}_{C}$ using non-holonomic constraints Eqs (3) shifted from the ball center $G$ to the contact point $C$ :

$$
\begin{aligned}
& \dot{u}_{C x}=\omega_{y}\left(u_{C z}-R\right)-\omega_{z} u_{C y}, \\
& \dot{u}_{C y}=\omega_{z} u_{C x}-\omega_{x}\left(u_{C z}-R\right), \\
& \dot{u}_{C z}=\omega_{x} u_{C y}-\omega_{y} u_{C x} .
\end{aligned}
$$

So we have the system of six non-linear $\operatorname{ODEs}$ Eqs $(4,5)$ in normal form with six unknowns functions of time: $u_{C x}, u_{C y}, u_{C z}, \omega_{x}, \omega_{y}, \omega_{z}$. Vector $\mathbf{u}_{C}$ depict displacements of the contact point and can be used to study the trajectory of the ball from the global point of view. Detailed behavior of the ball as a rotating body is given by angular velocities $\omega$. If the time history of rotation should be traced, then a subsequent run is necessary to obtain rotations (by means of Euler angles) as a solution of the system of three ODEs with an input of angular velocities $\omega$, see e.g. [2, 3].

It is worth to emphasize that the system Eqs $(4,5)$ has a significantly expressed autoparametric character, see e.g. [8]. Hence, semi-trivial solutions (STS) should be expected especially among singular solutions.

\section{Ball trajectories within the cavity}

A large program of a ball trajectory investigation within a spherical cavity has been performed using the differential system Eqs $(5,4)$. Basically it consists of two groups which are briefly illustrated in this and the next subsections. The first group concerns the fixed cavity (no excitation is applied). The only source of energy introduced is given by an initial deflection of the ball from equilibrium position in the point $A$ ("southern pole"), or in other words by the non-homogeneous initial conditions.

Differential system Eqs $(5,4)$ admits a number of singular solutions which can serve as separating limits of zones within which regular solutions exhibit certain character of trajectory shape. Some of them can be found analytically from the differential system taking into 


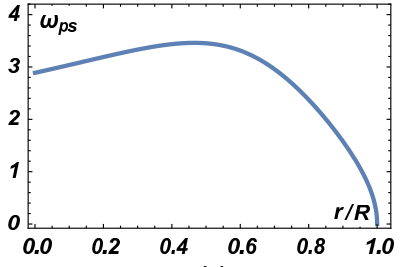

(a)

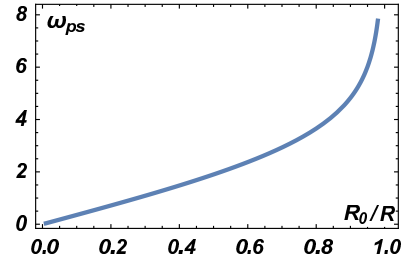

(b)

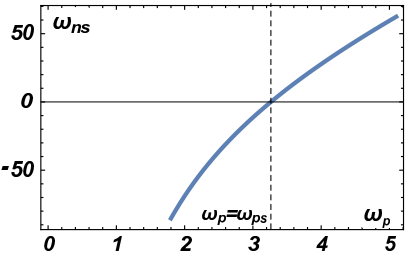

(c)

Figure 2. Initial velocity $\omega_{p s}$ producing the Separating Circle

account their special properties concerning individual response component along the trajectory as a whole or in certain points of these curves. For details special papers should be referred. Take a note, that most of the special cases emerge when no damping is considered. The reason is that the trajectory should be quasi-periodic (or cyclic-stationary), which is impossible when damping is respected and no stationary external energy supply is considered.

Trajectory starts in the point $C$ on the cavity meridian into which the ball is elevated (height is $u_{C z 0}$ and distance from the vertical axis is $R_{0}$. Then it is thrown horizontally along the cavity parallel circle employing circular velocity $\omega_{p}$, see Fig. 1. Four series of simulations relied on analytical support have been performed, two of them without damping and two respecting rolling and spinning damping, see Figs 3 and 4. Singular solutions regard damping free setting and therefore, we focus on this case and only a small demonstration is devoted to setting with damping, see Fig. 5.

These figures are organized in triplets following the initial setting. In every triplet: movement time history of the contact point $C: u_{C x}, u_{C y}, u_{C z}$; vertical view of the trajectory components $u_{C x}, u_{C y}$; axonometric view of the trajectory; parameters above the triplet: initial values of circular velocity vector $\omega_{0}=\left(\omega_{p 0}, \omega_{q 0}, \omega_{n 0}\right)$ and damping parameters $\kappa_{r}, \kappa_{s}$.

Let us briefly discuss a few of the most important singular (highlighted in gray in figures) and some intermediate solutions.

For the first series, no initial spin is considered $\left(\omega_{n 0}=0\right)$, see Fig. 3. Looking through the time history of all demonstrated settings, we can see that the response has the mono-harmonic character with slowly changing amplitudes and, therefore, these cases can be analytically investigated using the simple version of the harmonic balance method, see e.g. [9] and others.

(a) Circular trajectory in horizontal plane. See Fig. 3(al). This case is the most important and can be called Separating Circle (SC). The impulse applied corresponds with the initial velocity $\omega=\left(\omega_{p s}, 0,0\right)$, where it holds for $\omega_{p s}$ :

$$
\omega_{p s}=\frac{g M \rho u_{C z 0}\left(2 R-u_{C z 0}\right)}{\left(J+M \rho^{2} R^{2}\right)\left(R-u_{C z 0}\right)}, \quad R_{0}=\sqrt{R^{2}-\left(R-u_{C z 0}\right)^{2}}
$$

Fig. 2 plot (a) shows initial velocity $\omega_{p s}$ as a function of the ratio $r / R$ if $R_{0} / R=0.25$, while plot (b) regards $\omega_{p s}$ as a function of $R_{0} / R$ if $r / R=0.25$. Nevertheless, a small perturbation in $\omega_{p s}$ can be compensated by non-zero spin velocity $\omega_{n s} \neq 0$ and the pure circular motion can be kept, see plot (c) in Fig. 2:

$$
\omega_{n s}=\frac{g M \rho u_{C z 0}\left(u_{C z 0}-2 R\right)+\omega_{p}^{2}\left(R-u_{C z 0}\right)\left(J+M \rho^{2} R^{2}\right)}{J \omega_{p} \sqrt{u_{C z 0}\left(2 R-u_{C z 0}\right)}} .
$$

Regard limit states are: $\omega_{p}=\omega_{p s} \Rightarrow \omega_{n s}=0$ - basic case of SC, while next two cases reflect perturbed states $\omega_{n s} \neq 0: \omega_{p}<\omega_{p s} \Rightarrow \omega_{n s} \rightarrow+\infty \omega_{p}=0 ; \omega_{p}>\omega_{p s} \Rightarrow \omega_{n s} \rightarrow-\infty \omega_{p}=0$.

(b) Circular trajectory in inclined plane, see Figs 3(bl) and (cl), $\omega_{0}=[100.0,0,0]$. The planar trajectory exactly takes place state when $\omega_{p 0} \rightarrow \infty$. The space spiral type trajectory 
(I)

(a)

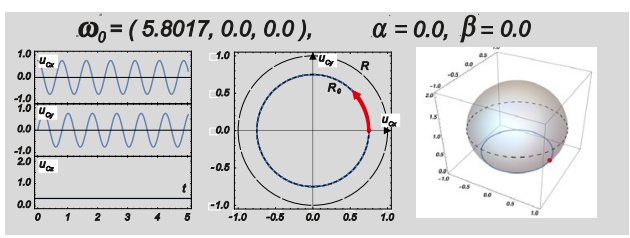

(b)
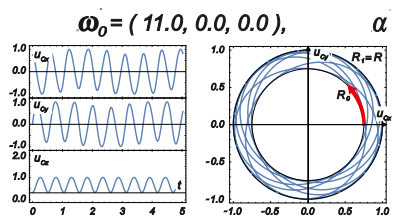

$\alpha=0.0, \beta=0.0$

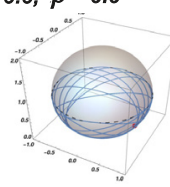

(c)

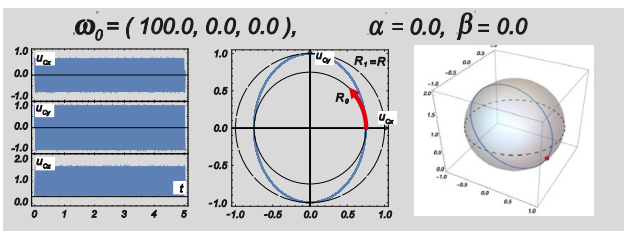

$(r)$
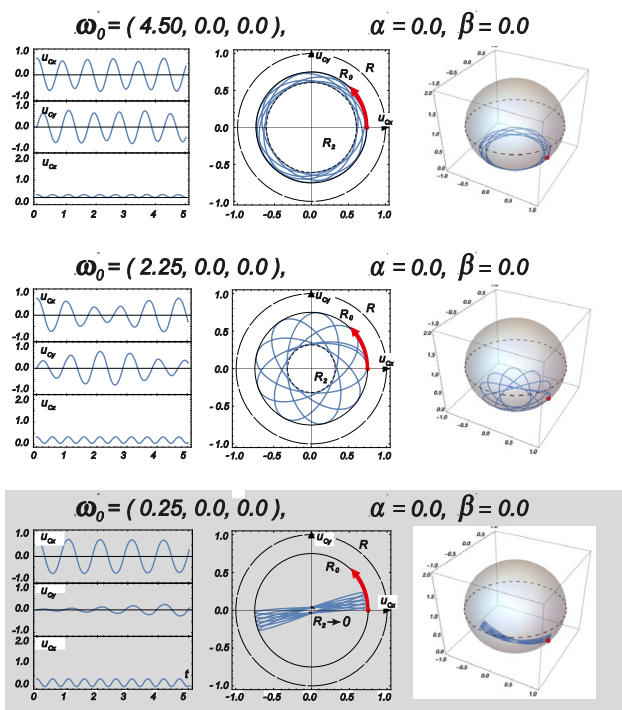

Figure 3. Illustration of the ball trajectories; initial spin $\omega_{n 0}$ and damping $\kappa_{r}, \kappa_{s}$ are not considered; left column $\omega_{p s} \leq \omega_{p}<+\infty$, right column $0 \leq \omega_{p}<\omega_{p s}$.

changes from SC upwards, see Fig. 3(bl). Its upper envelope is a circle with radius $R_{1}>R_{0}$, which rises until $R$ is reached. Then the spiral passes into the upper hemisphere and $R_{1}$ decreases back to $R_{0}$. Before the limit state for $\omega_{p 0} \rightarrow \infty$ is reached, the osculation plane of the trajectory can be recognized. It rotates around the vertical axis with descending angular velocity until that vanishes and osculation and operating planes coincide and $R_{1}=R_{0}$. The plane passes through the starting point $C_{0}$ and axis parallel with $y$ axis in the height $R$.

(c) Planar trajectory in vertical plane, see Fig. $3(\mathrm{cr}), \omega_{0}=[0.25,0,0]$. The right column demonstrates cases when $\omega_{p}<\omega_{p s}$. The spiral trajectory finds inside the SC. Its bottom level is given by the envelope circle with the radius $R_{2}<R_{0}$. As $\omega_{p} \rightarrow 0$ also $R_{2} \rightarrow 0$, see pictures (ar) and (br). The trajectory plotted in picture (cr) approaches the trajectory in vertical plane emerging for $\omega_{p}=0$. The state exhibited remembers the Foucault pendulum trajectory moving in a vertical osculation plane, which slowly rotates around the vertical axis $z$.

Let us get to the second series considering the initial spin of the ball, i.e. $\omega_{n 0} \neq 0$, see Fig. 4. Both sub-series for $0<\omega_{n 0}<+\infty$ and $-\infty<\omega_{n 0}<0$ starts from SC setting, see Fig. 3(al). Observing time histories again we conclude, that the response has the bi-harmonic character generally with irrational ratio of frequencies. Therefore, when analytically examined, the harmonic balance method can be still employed, but two independent harmonic components with slowly variable amplitudes should be considered, see e.g. [9] and others. May be except a small neighborhood of $\omega_{n 0}=0$, see Figs 4(al) and (ar).

(d) Trajectory of "kings crown form", see Fig. 4(bl), $\omega_{0}=[5.817,0,5.0537]$. For a special value of $\omega_{n 0}=\omega_{n 0-\text { lim }}=5.0537$ the trajectory takes a shape visible in the picture. The sharp apexes of this curve corresponds to $\omega=\left[0,0, \omega_{n 0}\right]$ and $\mathbf{u}=[0,0,0]$, which is a clue to find form and parameters of this singular case. If $\omega_{n 0}<\omega_{n 0-\text { lim }}$, see Fig. 4(al), the trajectory has a spiral form limited by the circular envelope with radius $R_{1}>R$. The "kings crown" trajectory is reached when the initial spin velocity equals $\omega_{n 0-l i m}$. Radius $R_{1}$ is maximal, but still holds $R_{1}<R$. It means that under this regime the spiral cannot pass into the upper hemisphere. For $\omega_{n 0}>\omega_{n 0-\lim }$ the trajectory assumes a curly shape, see Fig. 4(cl). Frequency of curls increases and their amplitude and radius $R_{1}$ decrease back approaching $R_{0}$ from external side. 
(I)

(a)

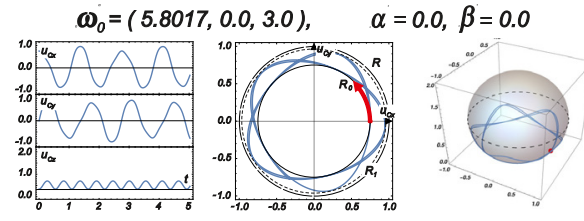

(b)

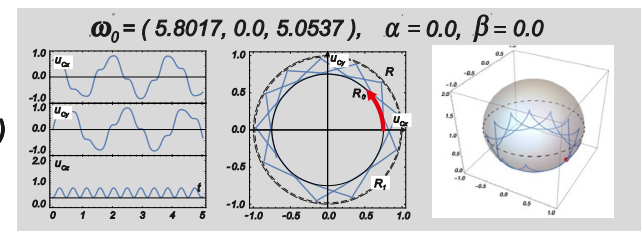

(c)
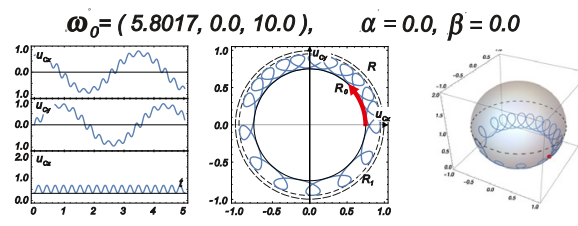

(d)

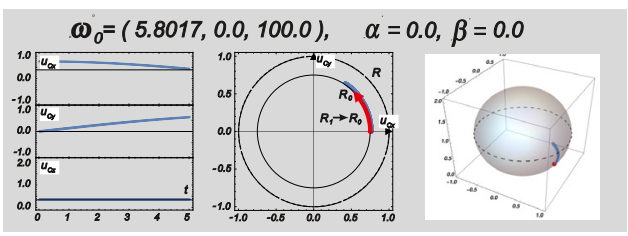

( $r)$
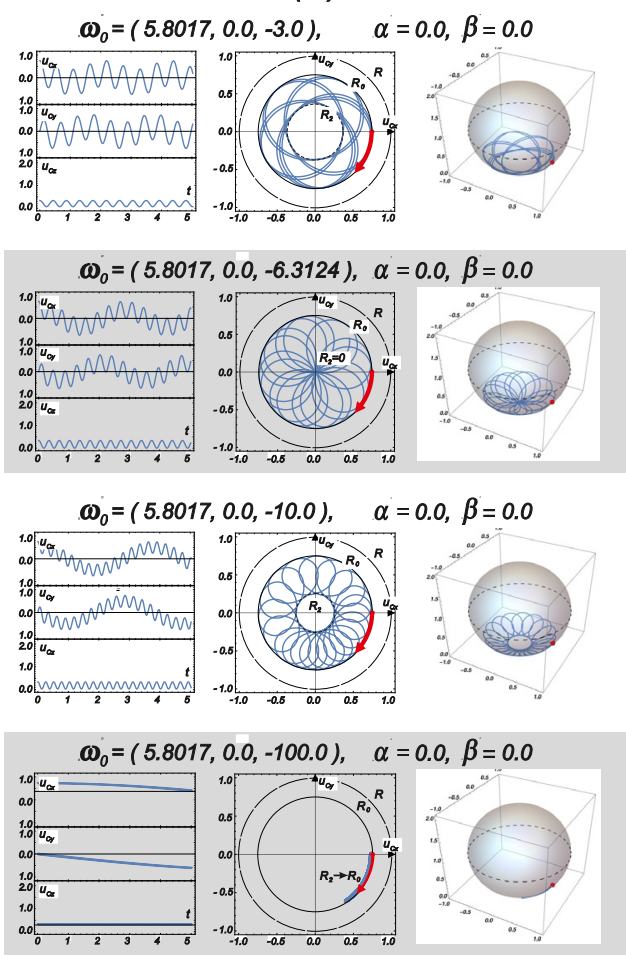

Figure 4. Illustration of the ball trajectories; damping $\kappa_{r}, \kappa_{s}$ is not considered; left column $\omega_{p}=\omega_{p s}, 0<$ $\omega_{n 0}<+\infty$, right column $\omega_{p}=\omega_{p s},-\infty<\omega_{n 0}<0$.

The effective velocity of the ball advancing along the contour line decreases and approaches zero.

(e) The limit state for infinite initial spin velocity: $\omega_{n 0} \rightarrow+\infty$, see Fig. $4(\mathrm{dl})$. The ball is apparently fixed in the initial point and it does not move neither horizontally nor vertically, although the initial impulse $\omega_{p 0}$ is applied.

(f) The trajectory with multi-point in A, see Fig. 4(br). This state corresponds with $\omega_{n 0-m p}=-6.3124<0$. Every loop of the spiral trajectory passes the point $A$. The envelope circle degenerates into point $A$ and $R_{2}=0$. For $\omega_{n 0-m p}<\omega_{n 0}<0$, see picture (ar), the trajectory has still rather a form of a simple spiral inside of SC. Every partial loop goes around the point $A$ and it is limited by the envelope circle with radius $R_{2}<R_{0}$. When $\omega_{n 0}$ descends below $\omega_{n 0-m p}$, i.e. $-\infty<\omega_{n 0}<\omega_{n 0-m p}<0$, the trajectory gets a curly form on internal side of SC and individual loops runs away the point $A$ with the envelope circle $R_{2}<R_{0}$. The frequency of curls rises with increasing $\omega_{n 0}$, while $R_{2}$ approaches $R_{0}$ or SC.

(g) The limit state analogous with ad(e): $\omega_{n 0} \rightarrow-\infty$, see Fig. 4(dr). The curly trajectory goes along the internal side of SC in the opposite direction as previously, picture (dl).

Let us have a look briefly at two pictures in Fig. 5. They respect the influence of damping. Coefficients $\kappa_{r}, \kappa_{s}$ are different as it corresponds with conditions in the real system. The left picture demonstrates trajectory for positive initial spin and the right for negative initial spin. The transition through limit cases mentioned above is visible. The trajectory obviously finishes in a small neighborhood of the bottom "southern pole" of the cavity. If the spin damping vanishes, we can find the ball still in rotating state, although its center almost does not move any more. 
(I)

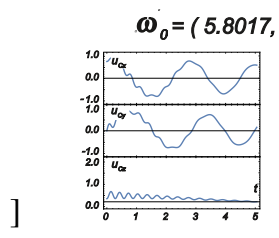

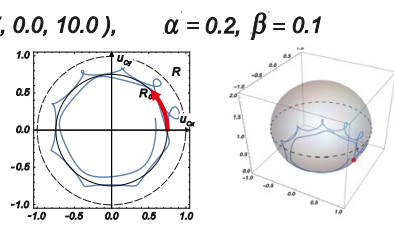

(r)

$\omega_{0}=(5.8017,0.0,-6.3210), \alpha=0.2, \beta=0.1$

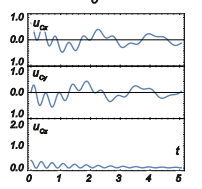

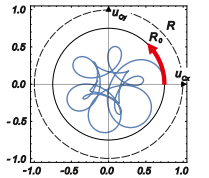

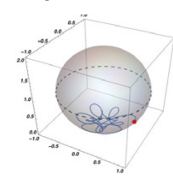

Figure 5. Two examples of the ball trajectories with considered damping; left picture $\omega_{n 0}>0$, right picture $\omega_{n 0}<0$.

\section{Conclusion}

The governing differential system modeling dynamic processes of a ball moving inside a spherical cavity has been presented. The system with six degrees of freedom has three nonholonomic constraints. Non-sliding contact between the ball and cavity is considered. Viscous damping of rolling and spinning movement is taken into account as energy loss in the system. The differential system has been deduced using the Appell-Gibbs approach. The full system has been thereafter discussed for fixed cavity concentrating to singular solutions of the homogeneous system with non-homogeneous initial conditions as the only source of energy injected into the system.

Seven singular solution types together with their neighborhood have been analyzed and physically interpreted. The set of singular solutions represent limits separating solution groups of a certain character. The shape and general character of regular solutions within individual domains delimited by these singular solutions are analyzed in order to facilitate a practical application of this theoretical background. As an example can be shown the trajectory, which occurs when one or both type of the damping is regarded.

In order to carry out analysis in a large scale of parameters, as frequencies, amplitudes, etc., no simplification of transcendent function using Taylor or other types of expansion has been adopted. Original form of all expressions is kept, which enables to obtain meaningful results also for extraordinary setting of parameters. Problems in related areas of non-holonomic systems as well as some hints for further study are outlined.

The kind support of the Czech Science Foundation project No. 17-26353J and of the RVO 68378297 institutional support are gratefully acknowledged.

\section{References}

[1] M. Pirner, O. Fischer, J IASS 41, 91-99 (2000)

[2] A. Lurie, Analytical Mechanics (in Russian). (GIFML, Moscow, 1961)

[3] L. Pars, A Treatise on Analytical Dynamics, 2 ed. (Ox Bow Press, Conn., USA, 1972)

[4] A. Lewis, Rep Math Phys 38, 11-28 (1996)

[5] E. Desloge, Am J Phys 56, 841-846 (1988)

[6] J. Náprstek, C. Fischer, in: A. Zingoni (Ed.), Insights and innovations in structural engineering, mechanics $\mathcal{F}$ computation (Taylor\& Francis, London, 2016) 214-219.

[7] J. Náprstek, C. Fischer, Procedia Engineer 199, 613-618 (2017)

[8] A. Tondl, T. Ruijgrok, F. Verhulst, R. Nabergoj, Autoparametric Resonance in Mechanical Systems (Cambridge University Press, Cambridge, 2000)

[9] Z. Xu, Y.K. Cheung, J Sound Vib 174, 563-576 (1994) 